\title{
Curcumin Alleviates LPS-Induced Inflammation and Apoptosis in Vascular Smooth Muscle Cells Via Inhibition of the NF- $\mathrm{K}$ B and JNK Signaling Pathways
}

Haohang Ruan ( $\sim$ Dr.haohangruan@hotmail.com )

Jiangxi Provincial People's Hospital https://orcid.org/0000-0002-7809-5009

Qing Huang

Jiangxi Provincial People's Hospital

Benting Wan

Jiangxi University of Finance and Economics

Ming Yang

Jiangxi Provincial People's Hospital

\section{Research Article}

Keywords: Curcumin, Lipopolysaccharide, vascular smooth muscle, NF- к B, JNK, atherosclerosis, inflammation

Posted Date: October 18th, 2021

DOI: https://doi.org/10.21203/rs.3.rs-969399/v1

License: (1) This work is licensed under a Creative Commons Attribution 4.0 International License.

Read Full License 


\section{Abstract}

Background: Curcumin plays an important role in inflammation. This study was aimed to investigate the effect of curcumin on vascular smooth muscle cells (VSMCs) injury induced by lipopolysaccharide (LPS) and its mechanism.

Methods: VSMCs cells were treated with different concentrations of curcumin $(0,50,100$ and $150 \mu \mathrm{g} /$ $\mathrm{mL}$ ). CCK-8 assay and flow cytometry were used to monitor the effects of curcumin on LPS-induced cell viability and apoptosis. The expression and release of inflammatory cytokines in VSMCs cells were detected by real-time quantitative polymerase chain reaction (qRT-PCR) and enzyme-linked immunosorbent assay (ELISA). The proteins expressions of NF- KB and JNK signaling pathways were analyzed by western blot.

Results: Curcumin could reduce LPS induced inflammatory injury by increasing VSMC's cell viability, reducing apoptosis and inhibiting the release of inflammatory cytokines. In addition, curcumin increased the expression of Toll-like receptor 4 (TLR4) in LPS treated VSMCs. In terms of mechanism, we found that curcumin attenuated LPS-induced cell injury in VSMCs via inhibition of NF- $\mathrm{K} B$ and the JNK signal pathway.

Conclusion: Curcumin can protect VSMCs from LPS induced inflammatory injury, which may be related to the blocking of NF- $\mathrm{k}$ B and the JNK signaling pathway. Curcumin may be a potential drug for the treatment of atherosclerosis.

\section{Introduction}

Atherosclerosis (AS) is the basic pathological basis of ischemic cardiovascular and cerebrovascular diseases [1]. Most scholars believe that AS is a chronic inflammatory reaction, and a variety of inflammatory factors and inflammatory cells participate in its occurrence and development. Recent studies suggest that endothelial damage is an important initiating factor in the formation of atherosclerotic plaque [2]. Vascular endothelial cells and smooth muscle cells are damaged by various risk factors, which leads to an excessive chronic inflammatory proliferative reaction in the local blood vessels [3]. Recent studies have reported that several Chinese herbal medicines can improve the symptoms of AS [4]. These findings have aroused people's interest in the treatment of AS with traditional Chinese medicine.

Curcumin is a plant polyphenol extracted from Curcuma longa and other medicinal plants. It has antiinflammatory, anti-tumor, anti-oxidation and immunomodulatory effects [5], which are related to its regulation of some molecular targets, including pro-inflammatory cytokines, growth factors, factors involved in proliferation and apoptosis, adhesion molecules and transcription factors [6-9]. Especially in vascular cells, curcumin can inhibit the over expression of pro-inflammatory cytokines induced by oxidized low density lipoprotein (ox LDL) in VSMCs [10]. However, the molecular mechanism of curcumin inhibiting LPS induced inflammation in VSMCs remains unclear. 
The purpose of this study was to investigate the therapeutic effect of curcumin on AS. The inflammatory injury model of VSMCs cells induced by LPS was established. We found that curcumin could reduce the damage of VSMCs cells induced by LPS. The protective effect of curcumin on VSMCs cells may be through the inhibition of NF - KB and JNK signal pathways. It is suggested that curcumin may be a potential drug for the treatment of AS.

\section{Materials And Methods}

\subsection{Cell culture and treatment}

Rat cell line VSMCs was cultured in Dulbecco's modified Eagle medium ((DMEM; Gibco BRL,Gaithersburg, MD, USA) containing $10 \%\left(\mathrm{v} / \mathrm{v}\right.$ ) fetal bovine serum ( $\mathrm{FBS} \square \mathrm{Gibco}$ ) in $37^{\circ} \mathrm{C}, 5 \% \mathrm{CO}_{2}$ atmosphere. Curcumin and LPS are purchased from Sigma-Aldrich (St. Louis, Missouri, USA). Firstly, VSMCs cells were treated with $10 \mu$ M LPS for 12 hours to establish the injury model. Then VSMCs cells were treated with different concentrations of curcumin $(0,50,100,150 \mu \mathrm{g} / \mathrm{mL})$ for 24 hours.

\subsection{Cell viability assay}

After digestion, the cells in the logarithmic growth phase were inoculated into 96-well plates at the density of $5 \times 10^{3} / \mathrm{ml}$ for 24 hours, and the old culture was discarded. Curcumin of different concentrations was added to each experimental group for 24 hours. Each experimental group had 4 compound holes, and each well was cultured with MTT solution Sigma-Aldrich (St.Louis, MO) (concentration was $5 \mathrm{mg} / \mathrm{ml}$ ) for 4 hours. The supernatant was carefully discarded, and $200 \mu$ I DMSO, was added to each well to dissolve and dissolve precipitation. The absorbance value was read by the enzyme labeling instrument at the wavelength of $490 \mathrm{~nm}$.

\subsection{Apoptosis assay}

Cell apoptosis was detected by annexin V-FITC double staining. 24 hours after transfection, the cells were digested by trypsin, collected and inoculated in a 6-well plate, the cell density was adjusted to $2 \times 10^{6}$ cells per well, cultured for 24 hours, the supernatant was discarded, pre-cold PBS was washed twice, $1 \times$ Binding buffer was used to suspend cells. The cell suspension was added with $5 \mu L$ annexin V-FITC and $5 \mu \mathrm{I} \mathrm{PI}$, After mixing well, the cells were incubated at room temperature for 15 minutes, The apoptotic rate was detected by flow cytometry (Beckman Coulter, Fullerton, CA, USA) within 1 hour, and the data were analyzed by Flow Jo software (tree star, Ashland, or, USA).

\subsection{Enzyme-linked immunosorbent assay}

VSMCs were seeded in 24 well culture dish and treated with different methods for 24 hours. The culture supernatant was collected. The concentrations of inflammatory cytokines interleukin-6 (IL-6), interleukin$1 \beta$ (IL-1 $\beta$ ) and tumor necrosis factor- $a$ (TNF- $\alpha$ ) were measured by ELISA kit (TaKaRa, Dalian).

\subsection{Quantitative Real-time PCR}


According to the instructions of the manufacturer, total RNA was isolated from VSMCs cells using TRIzol reagent (Invitrogen). Reverse transcription was carried out through multiple transcription kits (Applied Biosystems, Foster, CA, USA). To analyze IL-6, IL-1 $\beta$ and TNF- $a$, SYBR green PCR kit (TaKaRa) was used to quantify the messenger RNA (mRNA) levels of IL-6, IL-1 $\beta$ and TNF- $\alpha$. $\beta$-actin was amplified as a control. The relative expression levels of IL-6, IL-1 $\beta$ and TNF- a were calculated by the $2^{-\triangle \Delta C T}$ method.

\subsection{Western blot}

The total proteins of different groups of cells were extracted by a protein extraction kit. The protein concentration was determined by BCA (Pierce, Appleton, WI, USA). Total proteins were separated by SDSPAGE electrophoresis with $50 \mu \mathrm{g}$ total proteins in each well. After electrophoresis for 2 hours, the membrane was transferred to the PVDF membrane (Millipore, Billerica, Ma, USA) by the wet method. The membrane was sealed with $5 \%$ skimmed milk powder for 1 hour, and the primary antibody anti-Bax (ab32503, 1:1000), anti-pro-Caspase3 (ab32150, 1:1000), anti cleaved-Caspase3 (ab32042, 1:1000), antiIL-6 (ab233706, 1:1000), anti IL-1 3 (ab216995, 1:1000), anti-TNF-a (ab183218, 1:1000), anti-p65 (ab32536, 1:1000), anti-toll-like receptor 4 (anti-TLR4, ab22048, 1:500), anti-p-p65 (ab31624, 1:1000), anti-IKBa (ab109300, 1:1000), anti-JNK (ab176645, 1:1000), anti-p-JNK (ab176645, 1:1000), anti-c-Jun (ab40766, 1:1000) and anti-p-c-Jun (ab32385, 1:1000), anti- $\beta$-actin(ab8226, 1:2000), all purchased from Abcam (Cambridge, UK) was incubated at $4{ }^{\circ} \mathrm{C}$. Overnight, In the next morning, TBST was used to rinse the membrane, horseradish peroxidase-labeled second antibody was added and incubated at $37^{\circ} \mathrm{C}$ for 1 h. ECL was used to develop and save the image. The optical density value was measured by quantity one.

\subsection{Statistical analysis}

The data of this study were expressed by mean \pm standard deviation (SD), and the statistical software SPSS 19.0 was used (IBM Analytics, New York, USA), $P<0.05$ was considered to be a statistically significant result. All experiments are repeated at least three times.

\section{Results}

\subsection{Curcumin attenuated LPS induced cell injury in VSMCs}

To investigate the effect of curcumin on the inflammatory injury of VSMCs cells induced by lipopolysaccharide (LPS), the cell viability was observed by CCK- 8 assay, and the apoptosis rate was determined by flow cytometry before and after curcumin treatment. Consistent with previous studies [11, 12], our results showed that LPS could inhibit the viability of VSMCs cells. Curcumin could increase the viability of VSMCs cells in a dose-dependent manner ( Figure 1(A)). Besides, the percentage of apoptotic cells in the LPS treatment group was significantly higher than that in the control group, but curcumin could inhibit the pro-apoptotic effect of LPS in a dose-dependent manner (Figure 1(B)). In order to further confirm our results, a western-blot was used to detect the protein expression levels of specific markers of apoptosis (Bax, Caspase-3 and Caspase-9). As shown in (Figures. 1C and D), the levels of Bax, Pro- 
Caspase-3 and Pro-Caspase-9 proteins in the LPS treatment group were significantly higher than those in the control group, but the promoting effect of LPS and curcumin combined treatment group was significantly decreased. Taken together, these data suggested that curcumin attenuates LPS-induced VSMCs cell injury in a dose-dependent manner by increasing cell viability and reducing apoptosis.

\subsection{Curcumin inhibited LPS-induced production of inflammatory cytokines in VSMCs cells}

QRT-PCR, western blot and ELISA assay were used to detect the effect of curcumin on the production of inflammatory cytokines TNF- $a$, IL-1 $\beta$ and IL- 6 in VSMCs cells induced by LPS. The results of qRT-PCR and western blot showed that the mRNA and protein levels of TNF- $a$, IL-1 $\beta$ and IL- 6 in VSMCs cells treated with LPS were significantly higher than those in the control group $(P<0.05)$. Whereas, the increased expression of TNF- $a, I L-1 \beta$ and IL- 6 induced by LPS decreased in a dose-dependent manner under curcumin treatment (Figure 2A\&B). The results of ELISA were consistent with the above results. Compared with the control group, the release of TNF-a, IL-1 $\beta$ and IL- 6 in the LPS treatment group was significantly increased, while the promoting effect of LPS and curcumin on the release of TNF- $a, I L-1 \beta$ and IL- 6 induced by LPS was decreased. The release of inflammatory cytokines decreased with the increase of curcumin concentration (Figure 2C-E). It was suggested that curcumin could inhibit the excessive secretion of TNF-a, IL-1 $\beta$ and IL-6 in VSMCs cells induced by LPS in a dose-dependent manner.

\subsection{Curcumin increased TLR4 expression in VSMCs cells treated with LPS}

To investigate the effect of curcumin on the expression of TLR4 in VSMCs cells, the protein level of TLR4 was detected by using curcumin $(50,100,150 \mu \mathrm{g} / \mathrm{ml})$ co-treated with LPS for 24 hours. The results showed that LPS significantly induced the expression of TLR4 in VSMCs cells, while curcumin significantly inhibited the expression of TLR4. These data suggested that curcumin could inhibit the expression of TLR4 in VSMCs cells, indicating that TLR4 might play an important role in the regulation of LPS-induced cell injury by curcumin (Figure 3).

\subsection{Curcumin relieved LPS induced VSMCs cell injury by inhibiting NF- $K$ B and JNK signaling pathways}

Next, we discussed the effect of curcumin on the regulation of NF- $\mathrm{K} B$ and the JNK signal pathway. Curcumin $(50,100,150 \mu \mathrm{g} / \mathrm{ml})$ was co-treated with LPS for 24 hours. The protein levels of NF- $\mathrm{K} B$ and the JNK signal pathway were detected by western blot. Western blot results showed that LPS treatment increased the expression of p-p65, p-ІкBa, p-JNK and p-c-Jun, indicating that LPS could promote the signal transduction of NF- KB and JNK pathways. Under the action of curcumin, the expressions of $p-p 65$, $\mathrm{p}-\mathrm{K} \mathrm{Ba}, \mathrm{p}$-JNK and p-c-Jun were down-regulated in a dose-dependent manner, suggesting that curcumin could inhibit NF-KB and JNK signal pathways. In a word, curcumin attenuated LPS induced VSMCs cell injury by inhibiting NF- $\mathrm{K}$ B and JNK signal pathways (Figure A\&B). 


\section{Discussion}

AS is the basic pathological basis of coronary heart disease. At present, it is considered that lipid metabolism disorder, oxidative stress and inflammatory reaction are important factors in the occurrence and development of AS [13-15]. A clinical trial has been confirmed that anti-inflammatory intervention is more accurate and personalized in patients with coronary heart disease [16]. In this study, the LPSinduced VSMCs model was used to explore the effects of curcumin on apoptosis and inflammation of VSMCs cells. We found that curcumin could reduce the inflammatory injury induced by LPS by increasing the viability of VSMCs cells, reducing apoptosis and inhibiting the release of inflammatory cytokines. On the other hand, we found that curcumin increased the expression of toll-like receptor 4 (TLR4) in LPStreated VSMCs cells. In terms of mechanism, we found that curcumin attenuated LPS induced VSMCs cell injury by inhibiting NF- $\mathrm{K}$ B and JNK signal pathways. Therefore, it is suggested that diosgenin can inhibit the progress of AS.

Curcumin and its analogs have long been used to treat various diseases, and modern science has also provided a reliable scientific basis for the pharmacological effects of curcumin. A large number of studies have confirmed that curcumin has anti-inflammatory, antioxidant and other pharmacological effects by regulating various targeting factors $[17,18]$. There are more and more studies on curcumin in cardiovascular diseases. For instance, curcumin can be used to prevent neointimal hyperplasia after angioplasty and regulate the proliferation and migration of VSMCs through anti-inflammatory and antioxidant effects [19]. Meng et a/ have shown that curcumin through ROS related TLR4-MAPK /NF- KB pathway inhibits LPS induced inflammation in rat vascular smooth muscle cells in vitro [20]. On the other hand, curcumin decreased the expression of NF- KBp65 and DNA binding activity of NF- KB in VSMCs exposed to LPS [21]. AS is an important cause of coronary heart disease, cerebral infarction, peripheral vascular disease and other diseases, and is related to inflammation, oxidation and other factors. There are more and more studies on curcumin against AS. For example, curcumin may alleviate AS by inhibiting PI3K / AKT and JAK2 / STAT5 signal pathways [22]. Zhang et al have shown that curcumin inhibits the activation of NF-KB in macrophages and reduces the expression of TLR4 induced by LPS, thereby reducing the degree of AS lesion and inhibiting the development of AS [23]. In addition, more and more studies have shown that oxidative stress and inflammatory pathways are involved in the biological process of curcumin. For instance, curcumin inhibits osteogenic differentiation of human aortic valve interstitial cells by interfering with the activation of the NF-KB / AKT / ERK signaling pathway [24]. Wang et a/ have shown that curcumin inhibiting the JNK pathway can prevent diabetic cardiomyopathy [25]. Consistent with the above studies, we found that curcumin had a protective effect on VSMCs damage caused by LPS. In recent years, more and more evidence shows that curcumin can reduce the expression of TLR4, and TLR4 can recognize LPS and induce related signal pathways to play an important role in LPS [23]. Therefore, we further discussed the effect of curcumin on the expression of TLR4. We found that curcumin could significantly promote the expression of TLR4 in LPS-treated VSMCs cells. 
Moreover, we also studied the relevant signal pathways and revealed their potential mechanisms. NF-kB is an important signal pathway associated with many physiological functions, including cell proliferation, malignant transformation and inflammation [26]. Inhibition of this pathway can improve cell viability and reduce apoptosis and inflammatory injury. P65 and IkBa are key molecules of the NF-kB signaling pathway, and their phosphorylation usually increases apoptosis and inflammation [27]. Here, we found that the upregulation of $\mathrm{p}-\mathrm{p} 65$ and $\mathrm{p}-\mathrm{I} \mathrm{kBa}$ induced by LPS was inhibited by curcumin. Therefore, these data suggested that the alleviating effect of curcumin on inflammatory injury induced by LPS might be achieved by inhibiting the NF-KB signal pathway in VSMCs. Recent evidence shows that JNK signaling pathway is also related to cell proliferation, apoptosis and inflammation. JNK and its downstream molecule c-Jun are activated after injury [28]. In this study, western blot showed that the expression of pJNK and p-c-Jun increased in LPS treated group, but decreased after curcumin treatment. These data suggested that curcumin attenuated LPS-induced VSMCs cell injury by inhibiting NF-KB and JNK signaling pathways in VSMCs cells.

To sum up, curcumin can protect VSMCs cells from LPS-induced inflammatory injury by improving cell viability, reducing apoptosis and inhibiting the production of inflammatory cytokines. Curcumin may play a protective role by inhibiting NF-KB and JNK pathways. These results provide a basis for curcumin to be a potential drug for the treatment of AS. Our study may provide some reference for the treatment of AS.

\section{Declarations}

\section{Disclosure Statement}

No potential conflict of interest was reported by the authors.

\section{Funding}

Wan benting, Ruan haohang,etc.,research on health monitoring and chronic disease prevention service management system for urban and rural residents, 2016-7-2019, key R \& D project of Jiangxi Provincial Department of science and technology, the second undertaker.

\section{References}

1. Qi Kong, Xin Ma, Jun-Xuan Lyu, et al. Plasma RANTES level is correlated with cardio-cerebral atherosclerosis burden in patients with ischemic cerebrovascular disease. Chronic Dis Transl Med. 2020;6(1):46-54.

2. S Sitia, L Tomasoni, F Atzeni, et al. From endothelial dysfunction to atherosclerosis. Autoimmun Rev. 2010;9(12):830-4.

3. R-N Qu, W Qu. Metformin inhibits LPS-induced inflammatory response in VSMCs by regulating TLR4 and PPAR-y. Eur Rev Med Pharmacol Sci. 2019;23(11):4988-4995. 
4. Zhongyang Lu, Yanchun Li, Wing-Kin Syn, et al. Amitriptyline inhibits nonalcoholic steatohepatitis and atherosclerosis induced by high-fat diet and LPS through modulation of sphingolipid metabolism. Am J Physiol Endocrinol Metab. 2020;318(2):E131-E144.

5. Chuan Wang, Manabu Niimi, Teruo Watanabe, et al. Treatment of atherosclerosis by traditional Chinese medicine: Questions and quandaries. Atherosclerosis. 2018;277:136-144.

6. Hitomi Ozawa-Umeta, Atsuhiro Kishimoto, Atsushi Imaizumi, et al. Curcumin $\beta$-D-glucuronide exhibits anti-tumor effects on oxaliplatin-resistant colon cancer with less toxicity in vivo. Cancer Sci. 2020;111(5):1785-1793.

7. Bagher Farhood, Keywan Mortezaee, Nasser Hashemi Goradel, et al. Curcumin as an antiinflammatory agent: Implications to radiotherapy and chemotherapy. J Cell Physiol. 2019;234:57285740 .

8. Shameemah Abrahams, William L Haylett, Glynis Johnson, et al. Antioxidant effects of curcumin in models of neurodegeneration, aging, oxidative and nitrosative stress: A review. Neuroscience. 2019;406:1-21

9. Hamid Mollazadeh, Arrigo F G Cicero, Christopher N Blesso, et al. Immune modulation by curcumin: The role of interleukin-10. Crit Rev Food Sci Nutr. 2019;59:89-101.

10. Yi Zhong, Tingrong Liu, Zhigang Guo. Curcumin inhibits ox-LDL-induced MCP-1 expression by suppressing the p38MAPK and NF-KB pathways in rat vascular smooth muscle cells. Inflamm Res. 2012;61:61-7.

11. Mei-Hong Yu, Xi Li, Qian Li, et al. SAA1 increases NOX4/ROS production to promote LPS-induced inflammation in vascular smooth muscle cells through activating p38MAPK/NF-KB pathway. BMC Mol Cell Biol. 2019; 20(1): 15.

12. Felipe Bichi Strela, Bruna Ferro Brun, Rebeca Caldeira Machado Berger, et al. Lipopolysaccharide exposure modulates the contractile and migratory phenotypes of vascular smooth muscle cells. Life Sci. 2020;241:117098.

13. Shin-ya Morita. Metabolism and Modification of Apolipoprotein B-Containing Lipoproteins Involved in Dyslipidemia and Atherosclerosis. Biol Pharm Bull. 2016;39:1-24.

14. Ajoe John Kattoor, Naga Venkata K Pothineni, Deepak Palagiri, et al. Oxidative Stress in Atherosclerosis. Curr Atheroscler Rep. 2017;19:42.

15. Yuhua Zhu, Xuemei Xian, Zhenzhen Wang, et al. Research Progress on the Relationship between Atherosclerosis and Inflammation. Biomolecules. 2018;8:80.

16. Tong Liu, Chao Han, Lixian Sun, et al. Association between new circulating proinflammatory and anti-inflammatory adipocytokines with coronary artery disease. Coron Artery Dis. 2019;30:528-535.

17. Jiawei Zhang, Yaling Zheng, Yan Luo, et al. Curcumin inhibits LPS-induced neuroinflammation by promoting microglial M2 polarization via TREM2/ TLR4/ NF-KB pathways in BV2 cells. Mol Immunol. 2019;116:29-37.

18. Xinyu Lin, Dingping Bai, Zixi Wei, et al. Curcumin attenuates oxidative stress in RAW264.7 cells by increasing the activity of antioxidant enzymes and activating the Nrf2-Keap1 pathway. PLoS One. 
2019;14:e0216711.

19. Minghua Zhang, Yuntian Li, Hui Xie, et al. Curcumin inhibits proliferation, migration and neointimal formation of vascular smooth muscle via activating miR-22. Pharm Biol. 2020;58:610-619.

20. Zhe Meng, Chao Yan, Qian Deng, et al. Curcumin inhibits LPS-induced inflammation in rat vascular smooth muscle cells in vitro via ROS-relative TLR4-MAPK/NF-KB pathways. Acta Pharmacol Sin. 2013;34:901-11.

21. Yi Zhong, Jian Feng, Jiafu Li, et al. Curcumin prevents lipopolysaccharide-induced matrix metalloproteinase-2 activity via the Ras/MEK1/2 signaling pathway in rat vascular smooth muscle cells. Mol Med Rep. 2017;16:4315-4319.

22. Yezhou Li, Leilei Tian, Dajun Sun, et al. Curcumin ameliorates atherosclerosis through upregulation of miR-126. J Cell Physiolm. 2019;234:21049-21059.

23. Shanshan Zhang, Jun Zou, Peiyang Li, et al. Curcumin Protects against Atherosclerosis in Apolipoprotein E-Knockout Mice by Inhibiting Toll-like Receptor 4 Expression. J Agric Food Chem. 2018;66:449-456.

24. Tingwen Zhou, Yongjun Wang, Ming Liu, et al. Curcumin inhibits calcification of human aortic valve interstitial cells by interfering NF-KB, AKT, and ERK pathways. Phytother Res. 2020;34:2074-2081.

25. Yonggang Wang, Shanshan Zhou, Wanqing Sun, et al. Inhibition of JNK by novel curcumin analog C66 prevents diabetic cardiomyopathy with preservation of cardiac metallothionein expression. Am J Physiol Endocrinol Metab. 2014;306:E1239-47.

26. Xiaopeng Zhao, Lu Ma, Lu Dai, et al. TNF-a promotes the malignant transformation of intestinal stem cells through the NF-KB and Wnt/ $\beta$-catenin signaling pathways. Oncol Rep. 2020;44:577-588.

27. Xia Han, L U Zhang, Yingcai Liu, et al. Resveratrol protects H9c2 cells against hypoxia-induced apoptosis through miR-30d-5p/SIRT1/NF-kB axis. J Biosci. 2020;45:42.

28. Klaokwan Srisook, Sakulrat Mankhong, Natthakarn Chiranthanut, et al. Anti-inflammatory effect of trans-4-methoxycinnamaldehyde from Etlingera pavieana in LPS-stimulated macrophages mediated through inactivation of NF-KB and JNK/c-Jun signaling pathways and in rat models of acute inflammation. Toxicol Appl Pharmacol. 2019;371:3-11.

\section{Figures}



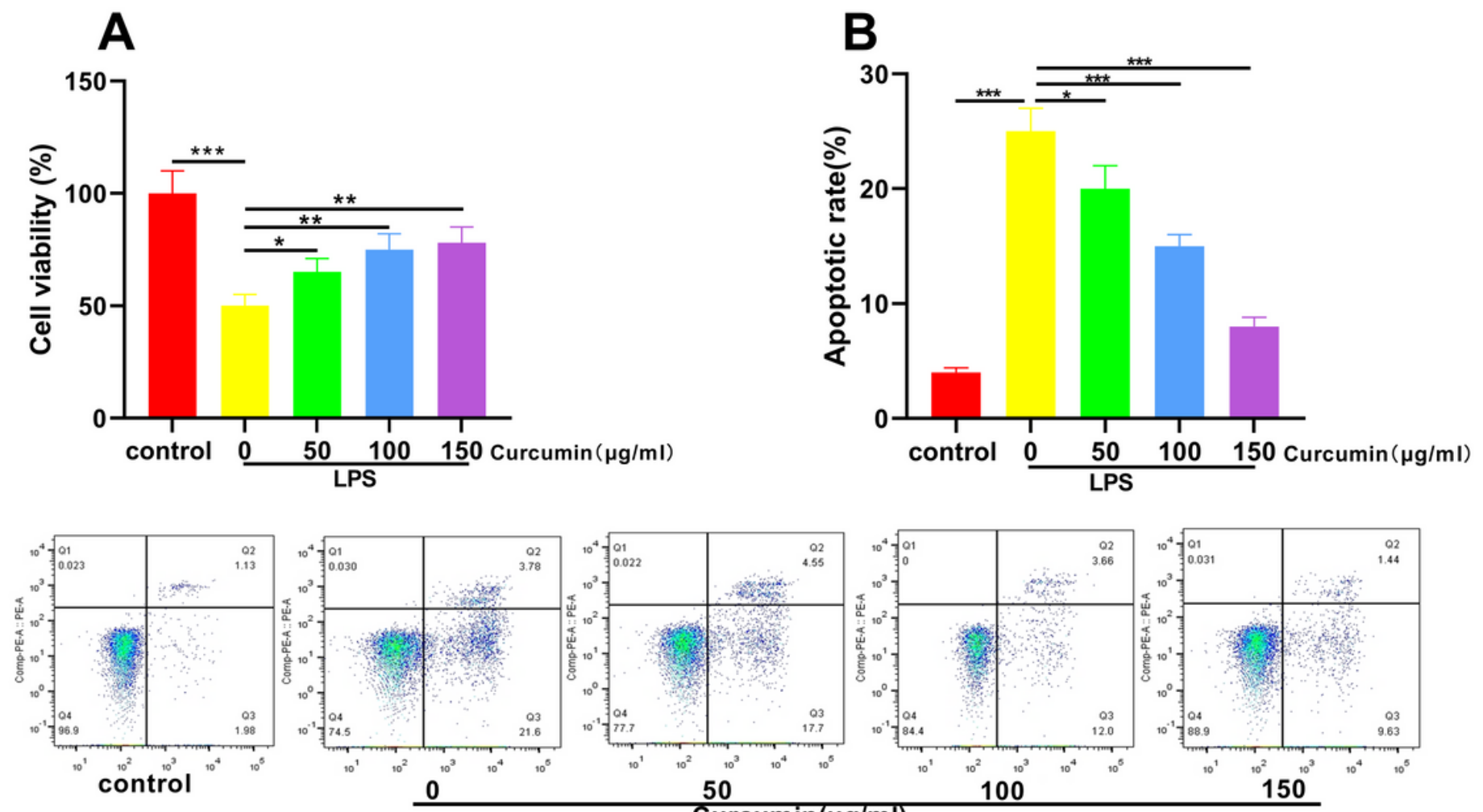

Curcumin $(\mu \mathrm{g} / \mathrm{mI})$
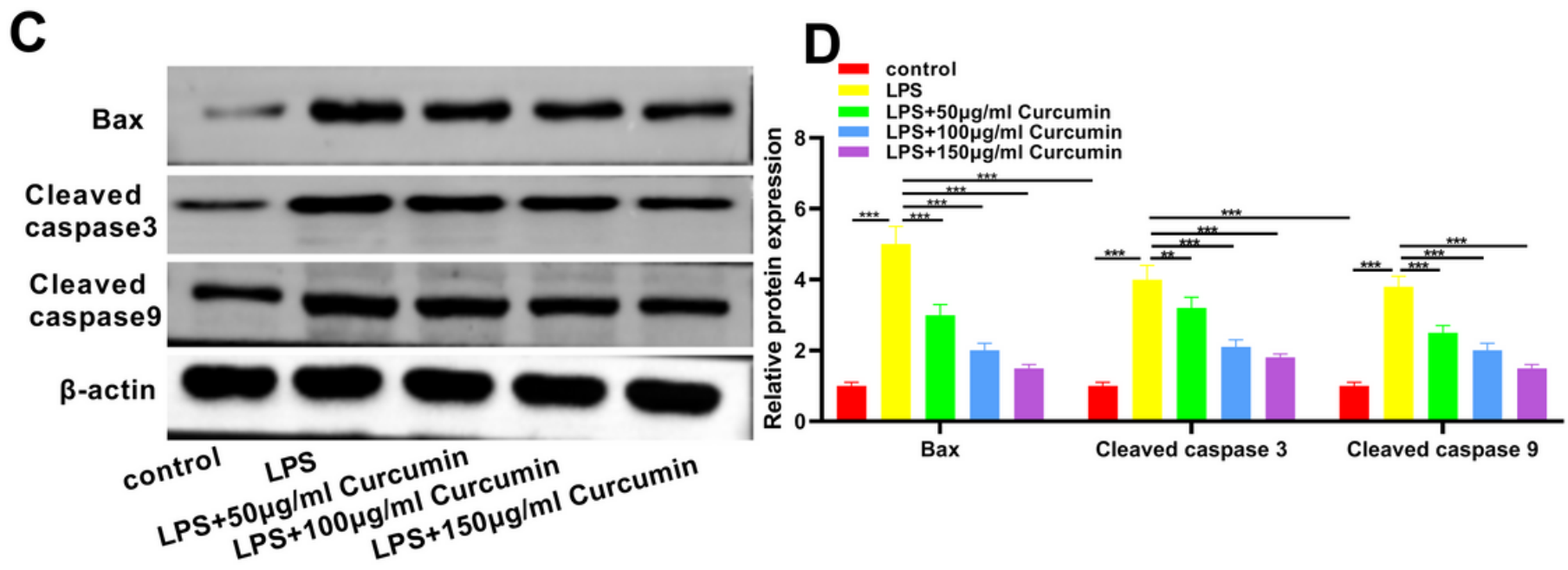

Figure 1

Curcumin attenuated LPS induced cell injury in VSMCs. The effects of curcumin $(0,50,100,150 \mu \mathrm{g} / \mathrm{ml})$ on LPS-induced VSMCs. (A)MTT assay was applied to detect cell viability. (B)Flow cytometry was used to monitor cell apoptosis. (C)western blot was applied to detect apoptosis the levels of related protein cleaved caspase3 and cleaved caspase $9 .{ }^{*}<<0.05 ;{ }^{*} P<0.01 ; * \star * P<0.001$. LPS, liposaccharide. 
A

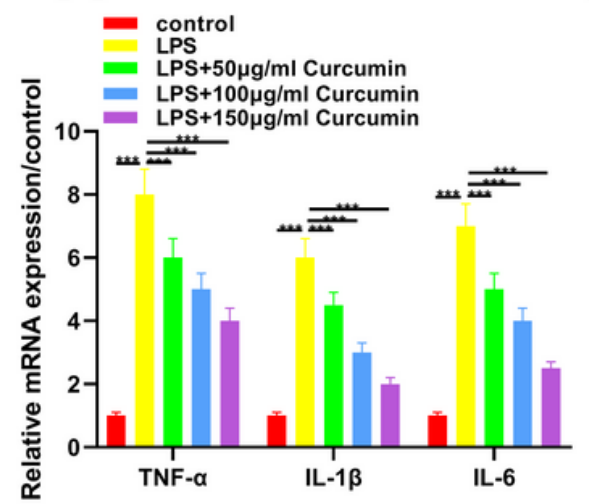

C

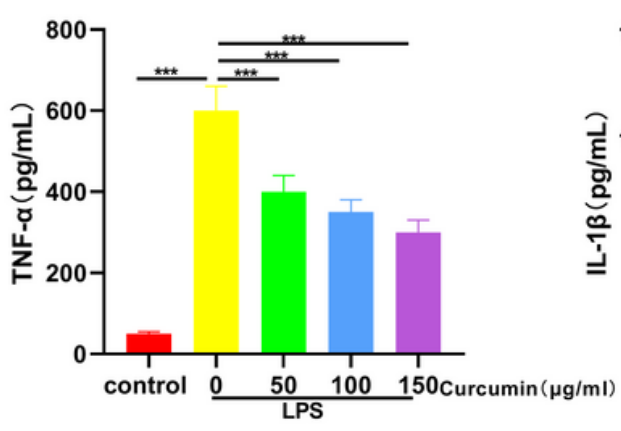

B
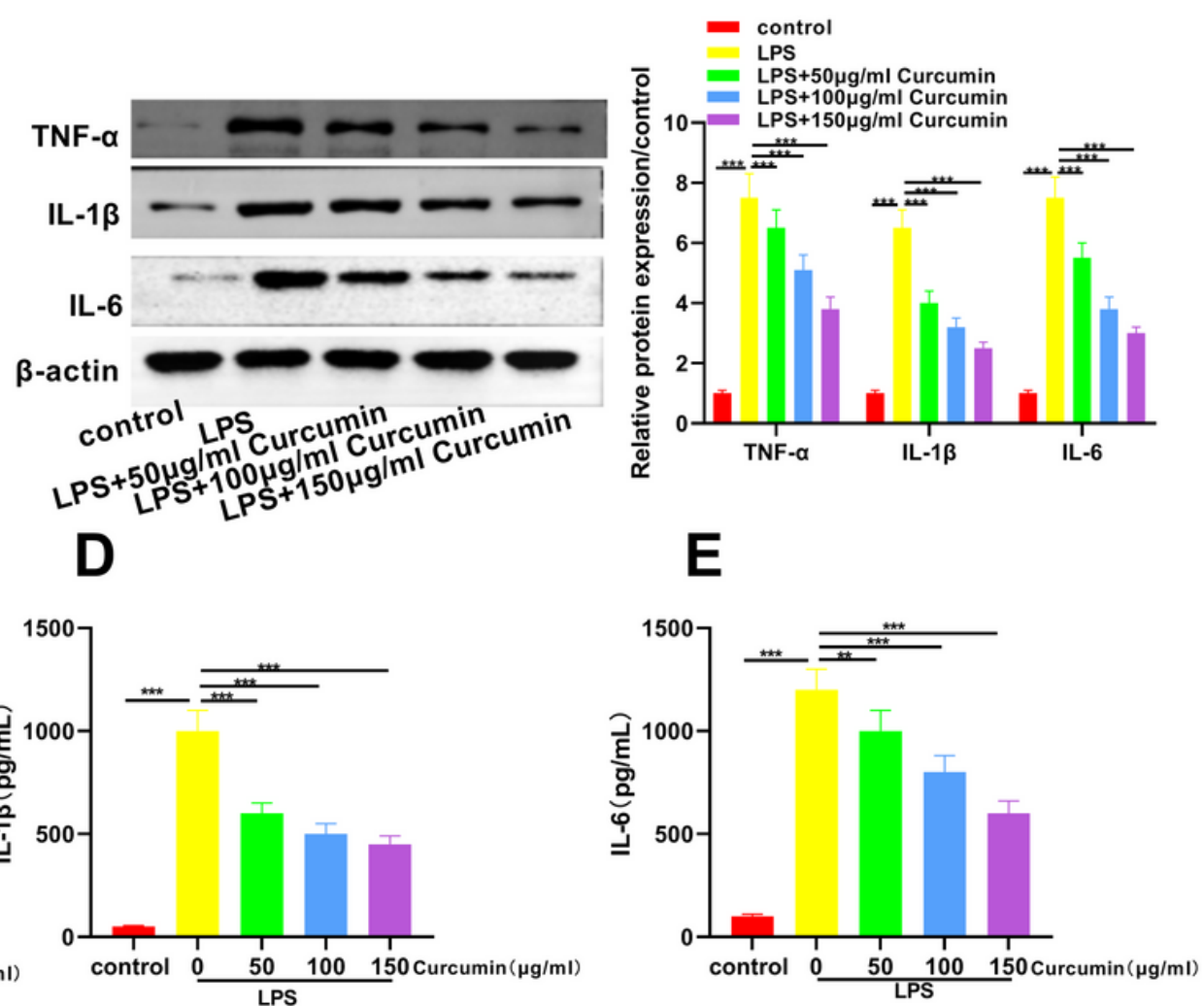

\section{Figure 2}

Curcumin inhibited LPS-induced production of inflammatory cytokines in VSMCs cells. The effects of curcumin $(0,50,100,150 \mu \mathrm{g} / \mathrm{ml})$ on LPS-induced VSMCs. (A)QRT-PCR was used to monitor the expressions of TNF-aXIL-1 $\beta$ and IL-6 mRNA. (B) western blot was applied to detect proteins level of TNF$\mathrm{a} \mathbb{\| I L - 1 \beta}$ and IL-6. (C) ELISA was used to detect the content of TNF-a冈IL-1 $\beta$ and IL-6 in VSMCs. ${ }^{\star * P}<0.01$; $\star \star * P<0.001$. LPS, lipopolysaccharide. 

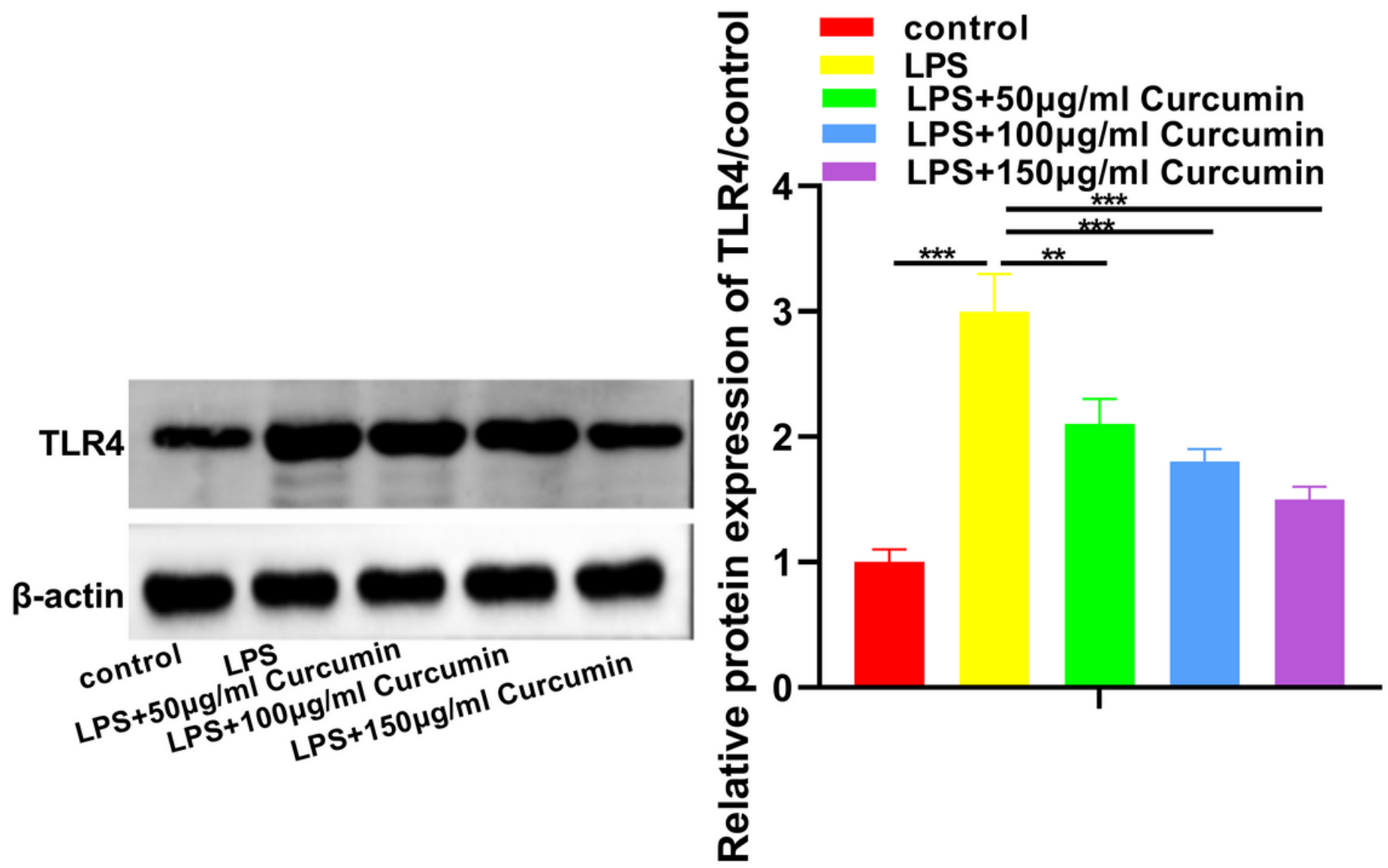

Figure 3

Curcumin increased TLR4 expression in VSMCs cells treated with LPS. The effects of curcumin $(0,50$, $100,150 \mu \mathrm{g} / \mathrm{ml}$ ) on LPS-induced VSMCs. Western blot was applied to detect the expression of TLR4. ${ }^{\star *} P<0.01 ;{ }^{* \star *} P<0.001$. LPS, lipopolysaccharide; TLR4, Toll-like receptor 4. 
A
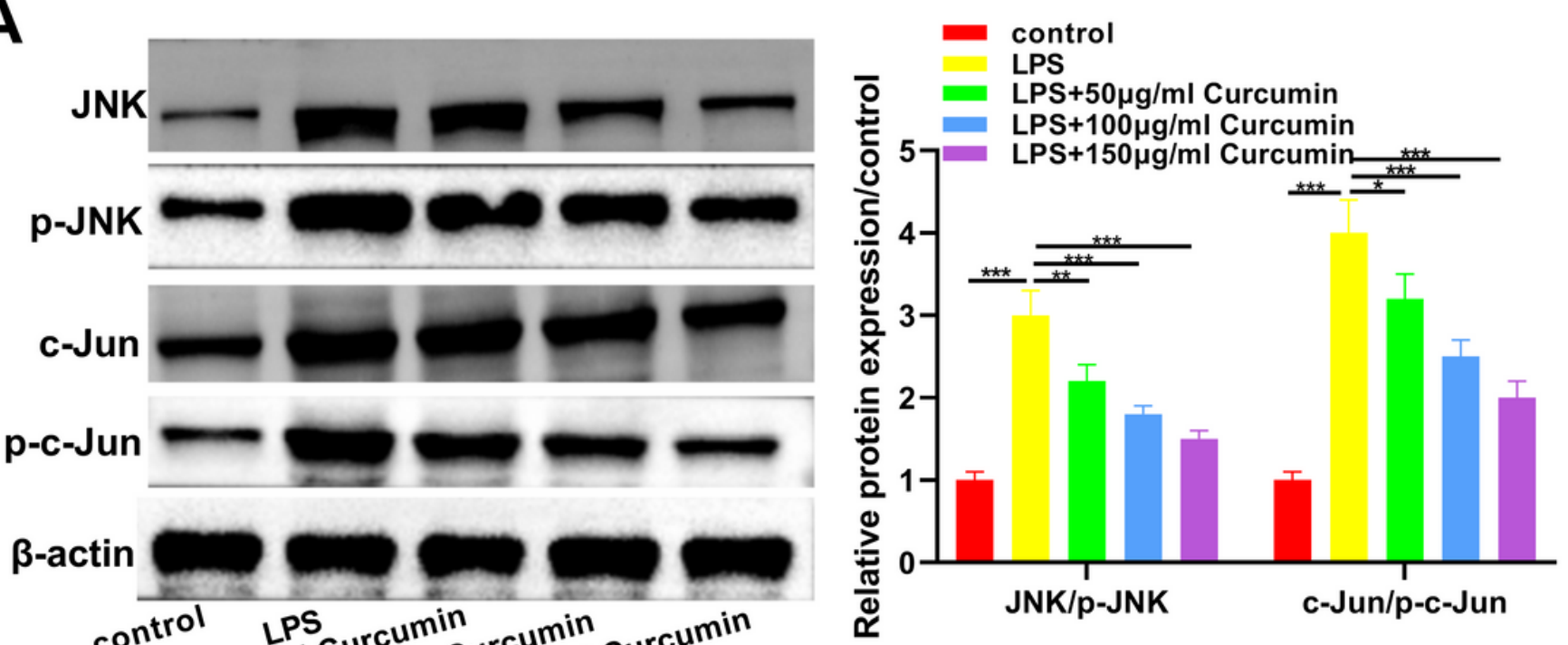

$\beta$-actin
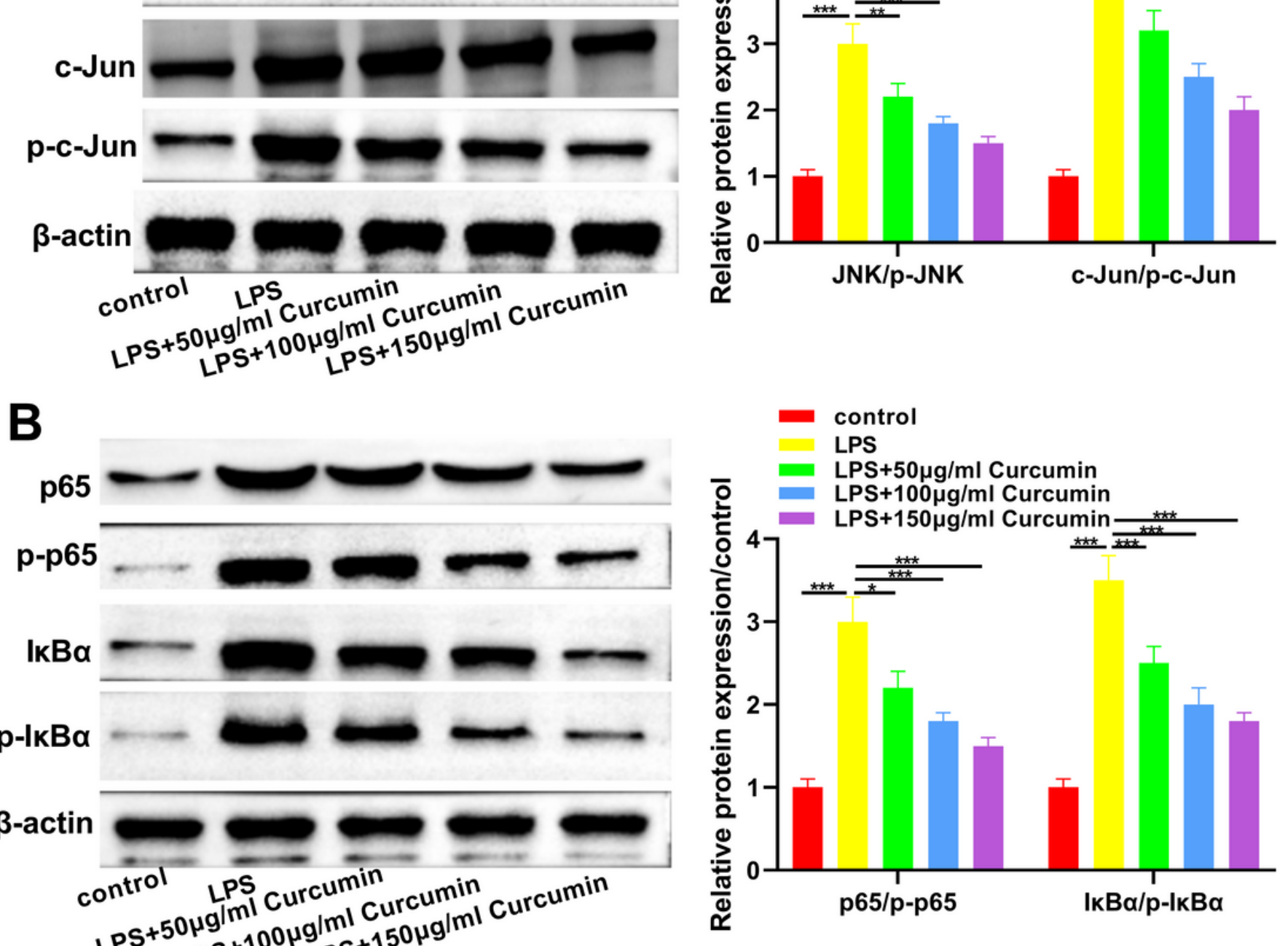

\section{Figure 4}

Curcumin relieved LPS induced VSMCs cell injury by inhibiting NF-KB and JNK signaling pathways. The effects of curcumin $(0,50,100,150 \mu \mathrm{g} / \mathrm{ml})$ on LPS-induced VSMCs. (A, B) western blot was applied to detect the expressions of JNK / p-JNK, Jun / p-Jun, p65 / p-p65, ІKBa / p-ІKBa. *P<0.05; **P<0.01; $\star \star \star P<0.001$. LPS, lipopolysaccharide.

\section{Supplementary Files}

This is a list of supplementary files associated with this preprint. Click to download.

- Graphicalabstract.png 